\title{
Clustered massive star formation in molecular clouds
}

\author{
Jonathan C. Tan \\ Inst. of Astronomy, Dept. of Physics, ETH Zürich, 8093 Zürich, Switzerland \\ email: jt@phys.ethz.ch
}

\begin{abstract}
I review some important questions in the field of massive star formation: What are the initial conditions for proto star clusters and how do they arise? What are the initial conditions for individual massive star formation within star clusters? How do massive protostars accumulate their mass? I compare the Turbulent Core Model (McKee \& Tan 2003) to several nearby regions, including Orion KL. Here I also discuss the origin of BN's high proper motion.
\end{abstract}

Keywords. stars: formation, ISM: kinematics and dynamics

\section{Introduction}

Throughout cosmic history, massive stars have been the principal drivers of galactic evolution, energizing and enriching gas, regulating its collapse into further stellar generations or even ejecting it into intergalactic space. The importance of massive stars is extended by the realization that a substantial fraction of all stars, and perhaps planetary systems, form in star clusters (Lada \& Lada 2003), where proximity amplifies the influence of massive star feedback. The fueling of supermassive black holes, from the Milky Way's relatively small example to the giants powering quasars, is also likely to be influenced by massive star formation (Goodman \& Tan 2004; Thompson et al. 2005).

Given this importance, our lack of understanding of massive star formation is shocking. For example, there has been no clear consensus on even the basic formation mechanism, whether it be collapse from approximately stellar mass gas cores via accretion disks (e.g. McLaughlin \& Pudritz 1997; Osorio et al. 1999; Yorke \& Sonnhalter 2002; McKee \& Tan 2003, hereafter MT03), competitive accretion of ambient cluster gas (Bonnell et al. 2001; Bonnell, Vine, \& Bate 2004; Schmeja \& Klessen 2004), or, most radically, direct stellar collisions (Bonnell, Bate, \& Zinnecker 1998). Even amongst the more conventional core models, a vast range of parameters, such as formation timescale or accretion rate, have been discussed. Present-day massive stars appear to form exclusively within star clusters (de Wit et al. 2005), but the timescale for star cluster formation is debated does it take few (Elmegreen 2000) or many (Tan 2004a) dynamical times? The influence of feedback in setting both the stellar initial mass function, including its upper limit, and the efficiency of star formation in clusters, is uncertain. Observationally, the closest example of a massive protostar (i.e. a star undergoing active growth by accretion), in the Orion Kleinmann-Low (KL) region, is so confusing that it has been used in support of all the different models (Tan 2004b; Bally \& Zinnecker 2005). Even amongst proponents of conventional models with accretion disks, the orientation of this disk axis is debated between two models that are orthogonal to each other (Greenhill et al. 2003; Tan 2004b).

The reasons for this confusion are, on the theoretical side, the range of scales that must be analyzed or simulated, the complicated physics exhibited by a self-gravitating, magnetized, turbulent, optically thick, chemically-evolving medium, and the large and 
uncertain parameter space and boundary conditions for the models and simulations. Observationally, the problems include the relative rarity of massive protostars, their typically large distance from us, their high obscuration, and the crowding of their environments.

In this review, I focus first on the initial conditions of star clusters ( 2 ) and then of massive stars and their mode of accretion(§3). My basic conclusion is that a model of formation involving the collapse of gas clumps and cores in approximate pressure equilibrium with their surroundings and in approximate virial equilibrium, can explain both star cluster and massive star formation. I give some examples of how the model applies to individual, well-studied regions of massive star formation ( $\S 4$ ), focusing particularly on Orion KL. Here I also give an update on the discussion of the origin of the BecklinNeugebauer $(\mathrm{BN})$ object, which is prominent in this region. Due to lack of space, I do not discuss feedback in star clusters or the timescale of star cluster formation.

\section{The Initial Conditions for Star Cluster Formation}

Figure 1 reviews the masses, $M$, and mean surface densities, $\Sigma=M /\left(\pi R^{2}\right)$, of local star clusters and interstellar gas clouds. For convenience $\Sigma=1 \mathrm{~g} \mathrm{~cm}^{-2}$ corresponds to $4800 M_{\odot} \mathrm{pc}^{-2}, N_{\mathrm{H}}=4.3 \times 10^{23} \mathrm{~cm}^{-2}$ and $A_{V}=200 \mathrm{mag}$, for the local gas to dust ratio. Contours of constant radial size, $R$, and $\mathrm{H}$ number density, $n_{\mathrm{H}}=\rho / \mu=3 M /\left(4 \pi R^{3} \mu\right)$ where $\mu=2.34 \times 10^{-24} \mathrm{~g}$ is the mean mass per $\mathrm{H}$, are indicated. Density contours correspond to free-fall times, $t_{\mathrm{ff}}=\sqrt{3 \pi /(32 G \rho)}=1.38 \times 10^{6}\left(n_{\mathrm{H}} / 10^{3} \mathrm{~cm}^{-3}\right)^{-1 / 2} \mathrm{yr}$.

The presence of molecules allows gas to cool to low temperatures, $\sim 10 \mathrm{~K}$, effectively removing thermal pressure support. To survive the destructive local interstellar FUV radiation requires a total column of $N_{\mathrm{H}}=(0.4,2.8) \times 10^{21} \mathrm{~cm}^{-2}$ for $\mathrm{H}_{2}$ and $\mathrm{CO}$, respectively (McKee 1999). Giant molecular clouds (GMCs) have a constant column of $N_{\mathrm{H}} \simeq(1.5 \pm 0.3) \times 10^{22} \mathrm{~cm}^{-2}$ and typical masses $\sim 10^{5}-10^{6} M_{\odot}$ (Solomon et al. 1987). A sample of local ( $d \lesssim 3 \mathrm{kpc}$ ) infrared-dark clouds (IRDCs) have masses of a few $\times 10^{3}-10^{4} M_{\odot}$ and $\Sigma \sim 0.1 \mathrm{~g} \mathrm{~cm}^{-2}$ (Kirkland \& Tan, in prep.), about 3 times the GMC mean. Several IRDCs with higher surface densities, $\sim 0.5-50 \mathrm{~g} \mathrm{~cm}^{-2}$, have been reported by Carey et al. (1998). Star-forming clumps observed in the sub-mm by Mueller et al. (2002) have similar masses, but $\Sigma \sim 0.1-1 \mathrm{~g} \mathrm{~cm}^{-2}$. More revealed systems, e.g. the Orion Nebula Cluster (ONC), have similar properties. These considerations suggest that IRDCs, forming from GMCs, are the initial conditions for star clusters. It also appears that large masses can accumulate in IRDCs before the onset of significant star formation.

Galactic star clusters more massive than $\sim 10^{4} M_{\odot}$ are quite rare. The Arches and Quintuplet clusters (e.g. Kim et al. 2000) are examples in the Galactic center region. The most massive young clusters, so-called super star clusters, are often found in starburst environments, e.g. the Antennae galaxies (Mengel et al. 2001), and in some dwarf galaxies, e.g. NGC 5253 (Turner et al. 2000) and NGC 1569 (Gilbert \& Graham 2003).

All these high-mass star-forming regions appear to have a density of $n_{\mathrm{H}} \sim 2 \times 10^{5} \mathrm{~cm}^{-3}$, about that at which the cooling rate is a maximum (Larson 2005), and thus gravitational collapse is easiest. A spherical self-gravitating cloud in hydrostatic equilibrium with mean surface density $\Sigma$ and density profile $\rho \propto r^{-k_{\rho}}$ with $k_{\rho}=1.5$, similar to observed clumps, has a mean pressure of $4.3 \times 10^{8} \Sigma^{2} \mathrm{~K} \mathrm{~cm}^{-3}$ (MT03). Massive stars and star clusters form under pressures $\gtrsim 3 \times 10^{7} \mathrm{~K} \mathrm{~cm}^{-3}$, much higher than that of the local diffuse ISM, i.e. $2.8 \times 10^{4} \mathrm{~K} \mathrm{~cm}^{-3}$ (Boulares \& Cox 1990).

What causes a particular region of a GMC to form a star cluster? From Figure 1 we see that the surface density, pressures, and volume densities must increase by at least factors of 10, 100, and 1000, respectively. This occurs in only a small part of the GMC: typically only $\sim 1 \%$ of the mass is involved. Models for the cause of star formation can 


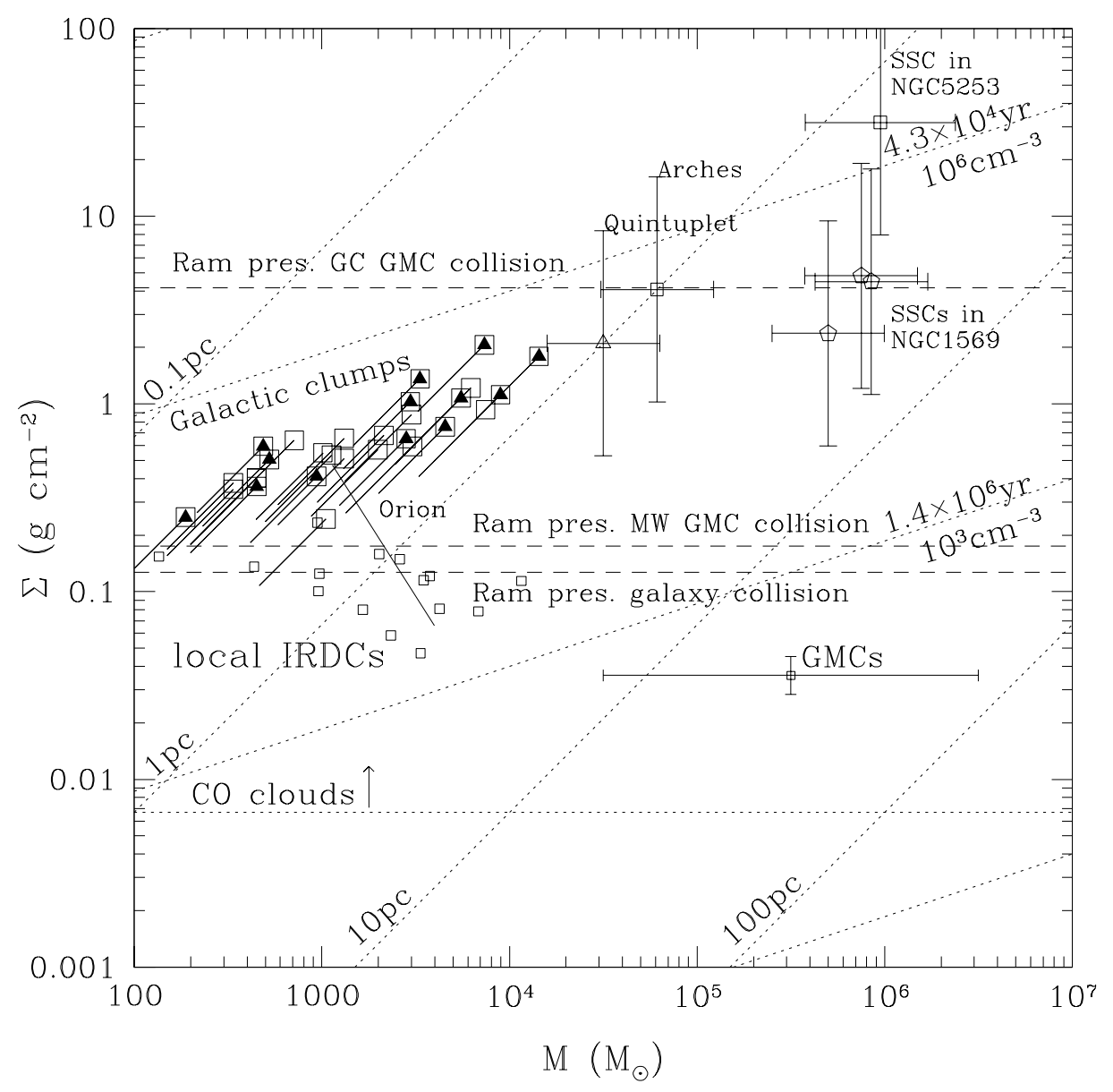

Figure 1. Surface density, $\Sigma$, versus mass, $M$, for star clusters and interstellar clouds. Contours of constant radius , $R$, and hydrogen number density, $n_{\mathrm{H}}$, or free-fall timescale, $t_{\mathrm{ff}}$, are shown with dotted lines. The minimum $\Sigma$ for CO clouds in the local Galactic FUV radiation field is shown, as are typical GMC parameters. Local infrared dark clouds are shown by small open squares (Kirkland \& Tan, in prep.). Large open squares are star-forming clumps (Mueller et al. 2002): a triangle indicates the clump contains an HII region; the diagonal lines show the effect of uncertain dust opacities on the mass estimate. The Orion Nebula Cluster, assuming equal mass in gas and stars, is shown by the solid line, which traces conditions from the inner to the outer parts of the cluster. Several more massive clusters are also indicated (see $\S 2$ ). Ram pressures from a typical Galactic GMC-GMC collision $\left(v=10 \mathrm{~km} \mathrm{~s}^{-1}, n_{\mathrm{H}}=500 \mathrm{~cm}^{-3}\right)$, a Galactic Center GMC-GMC collision $\left(v=40 \mathrm{~km} \mathrm{~s}^{-1}, n_{\mathrm{H}}=2.5 \times 10^{4} \mathrm{~cm}^{-3}\right)$, and in the diffuse ISM of a galaxy-galaxy collision $\left(v=200 \mathrm{~km} \mathrm{~s}^{-1}, n_{\mathrm{H}}=1 \mathrm{~cm}^{-3}\right)$ are indicated by horizontal dashed lines: note pressures are converted to an equivalent $\Sigma$ that would create the same mean pressure in a spherical, self-gravitating cloud, i.e. $P / k=4.3 \times 10^{8} \Sigma^{2} \mathrm{~K} \mathrm{~cm}^{-3}$ (MT03).

be divided into two groups: quiescent and triggered. In the former, star formation occurs in the densest, most unstable clumps of the GMC, and these form out of the general gravitational contraction of the entire cloud. This process may be regulated by the decay of turbulence, ambipolar (Mouschovias 1996) or turbulent diffusion of magnetic flux, or heating and ionization (McKee 1989) from newly-formed star clusters. 
In triggered models the star-forming clumps are created by compression of parts of the GMC by external causes, such as: cloud collisions (Scoville et al. 1986; Tan 2000); convergent turbulent flows (e.g. Mac Low \& Klessen 2004); or feedback from young stars with ionization (Elmegreen \& Lada 1977; Deharveng et al. 2005), stellar winds (e.g. Whitworth \& Francis 2002), protostellar outflows, radiation pressure, and supernovae (e.g. Palous et al. 1994). Elmegreen (2004) noted that compressions resulting from most forms of stellar feedback are probably only efficient within particular GMCs or GMC complexes, i.e. young stars forming in one GMC are unlikely to trigger star formation in another. Oey et al. (2005) claim the age sequence of 3 regions of the W3/W4 complex is evidence for triggered star formation over an approximately $100 \mathrm{pc}$ scale region.

Considering cloud collisions or convergent flows, the typical ram pressures, $P=\rho v^{2} \rightarrow$ $1.73 \times 10^{7}\left(n_{\mathrm{H}} / 1000 \mathrm{~cm}^{-3}\right)\left(v / 10 \mathrm{~km} \mathrm{~s}^{-1}\right)^{2} \mathrm{~K} \mathrm{~cm}^{-3}$, created in a variety of interactions are shown in Fig. 1. GMC collisions could compress local regions to pressures relevant to IRDCs. Pre-existing dense, stable clumps that become enveloped by a newly overpressured region would then start to collapse to form star clusters. Such a mechanism predicts a high degree of correlation in the locations of young star clusters within GMCs.

\section{The Initial Conditions and Mode of Accretion of Massive Stars}

Star clusters are born from turbulent gas, i.e. having velocity dispersions much greater than thermal. A basic question is how individual stars, particularly massive stars, form in this environment. Do they grow inside quasi-equilibrium gas cores that collapse via accretion disks with relatively stable orientations? In this scenario (e.g. Shu, Adams, \& Lizano 1987, MT03) the initial mass of the core helps to determine the final mass of the star, modulo the effects of protostellar feedback. Alternative models involving competitive Bondi-Hoyle accretion (Bonnell et al. 2001; Bonnell et al. 2004; Schmeja \& Klessen 2004) and direct stellar collisions (Bonnell, Bate, \& Zinnecker 1998) have been proposed. These models have been particularly motivated for massive star formation since this occurs in the most crowded regions, radiation pressure feedback from massive stars on dust grains can cause problems for standard accretion scenarios, and the Jeans mass in these high pressure, high density regions is only a fraction of a solar mass.

\subsection{Formation of Cores}

First consider the formation of cores from a turbulent medium. Klessen et al. (2005) find that a substantial fraction of "cores" identified in their nonmagnetic SPH simulations of supersonic turbulence appear quiescent (line widths $\leqslant$ thermal) and coherent (line widths roughly independent of positional offset from core center), but are in fact dynamic and transient. They argue that the inference of hydrostatic equilibrium, e.g. from radial profiles appearing similar to Bonnor-Ebert profiles (Alves, Lada, \& Lada 2001), is not necessarily valid, since such profiles are also possible for dynamically evolving cores. However, it is not clear if these artificial dynamic cores are consistent with the observations of Walsh et al. (2004), who find very small $\left(\lesssim 0.1 \mathrm{~km} \mathrm{~s}^{-1}\right)$ velocity differences between the line centers of high $\left(n_{\mathrm{H}} \sim 4 \times 10^{5} \mathrm{~cm}^{-3}\right)$ and low $\left(n_{\mathrm{H}} \sim 2 \times 10^{3} \mathrm{~cm}^{-3}\right)$ density traces of starless cores, i.e. these cores are not moving with respect to their envelopes.

The numerical simulations described above are nonmagnetic. Vázquez-Semadeni et al. (2005) studied core formation from turbulent, magnetized gas. They find in their periodic, fixed grid, isothermal, ideal MHD, driven turbulence simulations, that: magnetic fields reduce the probability of core formation; in the magnetically subcritical run, a bound core forms that lasts $\sim 5 t_{\mathrm{ff}}$ (defined at densities $\sim 50$ times the simulation mean), which is long enough for ambipolar diffusion to affect the dynamics; in the moderately supercritical 
case, where magnetic fields are relatively weaker, bound cores form and then undergo runaway collapse over about $2 t_{\mathrm{ff}}$, defined at the core's mean density. These results suggest that the initial conditions for star formation are bound cores, and that the stronger the magnetic field, the more chance cores have to attain a quasi-equilibrium structure. The marginally critical case is probably most relevant if star-forming clumps evolve from regions of GMCs that gradually lose magnetic support. Crutcher's (1999) observations of the magnetic field strength in dense regions of GMCs imply that these regions are only marginally supercritical and that magnetic fields are dynamically important.

Magnetic fields are likely to affect the mass function of cores. One argument against massive star formation from massive cores has been that the thermal Jeans mass in the high pressure, high density regions associated with massive star formation is very small. However this is irrelevant if massive cores derive most of their pressure support from either magnetic fields or turbulent motions. Observations suggest that the mass function of cores is fairly similar, within large uncertainties, to that of stars and that there are some massive pre-stellar cores (e.g. Beuther \& Schilke 2004).

\subsection{Accretion to Stars}

It is computationally expensive to follow gravitational collapse to the high densities and short timescales associated with protostars and their accretion disks. A common numerical technique is to introduce sink particles in bound regions of high density, which can then accrete gas from their surroundings (Bate, Bonnell, \& Price 1995).

Bonnell et al. (2004) modeled star cluster formation with SPH, isothermal, non-periodic, no feedback, nonmagnetic simulations, with initially turbulent gas (no later driving) and sink particles about to undergo global collapse. Stars gained mass by competitive accretion. The most massive star acquired gas that was initially very widely distributed. Dobbs, Bonnell, \& Clark (2005) found that a massive turbulent core, such as envisaged by MT03, fragments into many smaller cores if the equation of state is isothermal. However, their non-isothermal model suffered much less fragmentation.

We have seen that SPH simulations, by lacking magnetic fields, probably do not accurately model the fragmentation process of real star-forming regions, particularly with regard to core formation. Another difficulty is in resolving the accretion by sink particles of gas with vorticity (Krumholz, McKee, \& Klein 2005), with SPH simulations tending to greatly overestimate the accretion rate. Stellar feedback should also reduce this accretion rate, particularly to massive stars (e.g. Edgar \& Clarke 2004). Thus the importance of competitive accretion may be grossly overestimated in current SPH simulations.

\subsection{Assumptions and Predictions of the Turbulent Core Model}

McKee \& Tan (2002; 2003, MT03) modeled massive star formation by assuming an initial condition that is a marginally unstable, massive, turbulent core in approximate pressure equilibrium with the surrounding protocluster medium, i.e. the star-forming clump. To derive the pressure in the clump, it was also assumed to be in approximate hydrostatic equilibrium so that $P \sim G \Sigma^{2}$. This pressure sets the overall density normalization of each core and thus its collapse time and accretion rate.

The core is assumed to collapse via an accretion disk to form a single star or binary. The assumption of collapse in isolation is approximate: MT03 estimate that during the collapse the core interacts with a mass of ambient gas similar to its initial mass, although not all of this will become bound to the core. The core density structure adopted by MT03 is $\rho \propto r^{-k_{\rho}}$, with $k_{\rho}=1.5$ set from observations. This choice affects the evolution of the accretion rate: $k_{\rho}<2$ implies accretion rates accelerate. However, this is a secondary effect compared to the overall normalization of the accretion rate that is set by the 
external pressure. Also, since much of the pressure support is nonthermal with significant contributions from turbulent motions, one does not expect a smooth density distribution in the collapsing core, and the accretion rate can show large variations about the mean.

Some key predictions are the properties of the cores and accretion disks of massive stars. The core size is $R_{\text {core }} \simeq 0.06\left(M_{\text {core }} / 60 M_{\odot}\right)^{1 / 2} \Sigma^{-1 / 2}$ pc. Note an allowance has been made for massive cores tending to be near the centers of clumps, where pressures are about twice the mean (MT03). These cores have relatively small cross-sections for close interactions with other stars. The accretion rate to the star, via a disk, is $\dot{m}_{*}=$ $4.6 \times 10^{-4} f_{*}^{1 / 2} M_{60}^{3 / 4} \Sigma^{3 / 4} M_{\odot} \mathrm{yr}^{-1}$, where $f_{*}$ is the ratio of $m_{*}$ to the final stellar mass and a $50 \%$ formation efficiency due to protostellar outflows is assumed. The collapse time, $1.3 \times 10^{5} M_{60}^{1 / 4} \Sigma^{-3 / 4} \mathrm{yr}$, is short and quite insensitive to $M$, allowing coeval stochastic high and low mass star formation in a cluster, that might take $\gtrsim 1$ Myr to build up. The disk size is $R_{\text {disk }}=1200(\beta / 0.02)\left(f_{*} M_{60}\right)^{1 / 2} \Sigma^{-1 / 2}$ AU, where $\beta$ is the initial ratio of rotational to gravitational energy of the core, and the normalization is taken from typical low-mass cores (Goodman et al. 1993), although there is quite a large dispersion about this value. These estimates allow quantitative models of the protostellar evolution, disk structure and outflow intensity, which can then be compared to observed systems.

\section{Comparison of the Turbulent Core Model to Observed Regions}

We now compare the predicted properties of the turbulent core model (MT03) to some observed regions of massive star formation. The most basic comparison we can make is the size of the core with the scale of the region (Fig. 2).

\subsection{W49A}

W49A, $11.2 \mathrm{kpc}$ away, is one of the richest regions of massive star formation. Many ultracompact HII regions are seen in radio continuum maps and each one may be powered by a young $\mathrm{OB}$ star. In Fig. 2 we show the size of a $60 M_{\odot}$ core in pressure equilibrium with the central region of a $\Sigma=1 \mathrm{~g} \mathrm{~cm}^{-2}$ protocluster clump (adopted as a typical value — see Fig. 1 - and not based on a particular measurement of W49A). Fig. 2 also shows how far a core might move during its star formation timescale, $\sim 10^{5} \mathrm{yr}$, if traveling at the velocity dispersion of the system, taken to be $\sim 5 \mathrm{~km} \mathrm{~s}^{-1}$. We see that, while the region has a high density of massive stars, this density does not prevent massive stars from forming in relative isolation from each other, particularly allowing for projection effects. Of course interactions with lower-mass stars will be more common, but most of these would make only minor perturbations to the structure of the core.

\section{2. $W 3\left(\mathrm{H}_{2} \mathrm{O}\right)$}

$\mathrm{W} 3\left(\mathrm{H}_{2} \mathrm{O}\right)$ and $\mathrm{W} 3(\mathrm{OH})$ are much closer $(2.07 \mathrm{kpc}$, Hachisuka et al. 2004). While W3(OH) is an ultracompact HII region, probably powered by a massive star that has completed its formation, $\mathrm{W} 3\left(\mathrm{H}_{2} \mathrm{O}\right)$ is likely to be a massive protostar, i.e. still growing substantially by accretion. The luminosity of $\mathrm{W} 3\left(\mathrm{H}_{2} \mathrm{O}\right)$ is $\sim 1-9 \times 10^{4} L_{\odot}$ (Wyrowski et al. 1999). MT03 used this to estimate a current protostellar mass in the range $10-23 M_{\odot}$. Given that the star is still accreting and that Wyrowski et al. (1999) estimate a gas mass of $\sim 15 M_{\odot}$ for the inner $0.01 \mathrm{pc}$ by $0.02 \mathrm{pc}$ region, we again show the scale of a $60 M_{\odot}$ core that might eventually form a $30 M_{\odot}$ star. This scale is several times larger than the detected $1.3 \mathrm{~mm}$ emission and a few times larger than the $\mathrm{C}^{18} \mathrm{O}$ emission (Wyrowski et al. 1999). To reconcile this, one could consider somewhat lower mass cores in higher pressure environments. However, note that the cores shown in Fig. 2 represent the initial configuration before onset of collapse. At later stages the core becomes more concentrated. Also, there may be more core material beyond the outer contour of $\mathrm{C}^{18} \mathrm{O}$ emission shown by Wyrowski et al. 

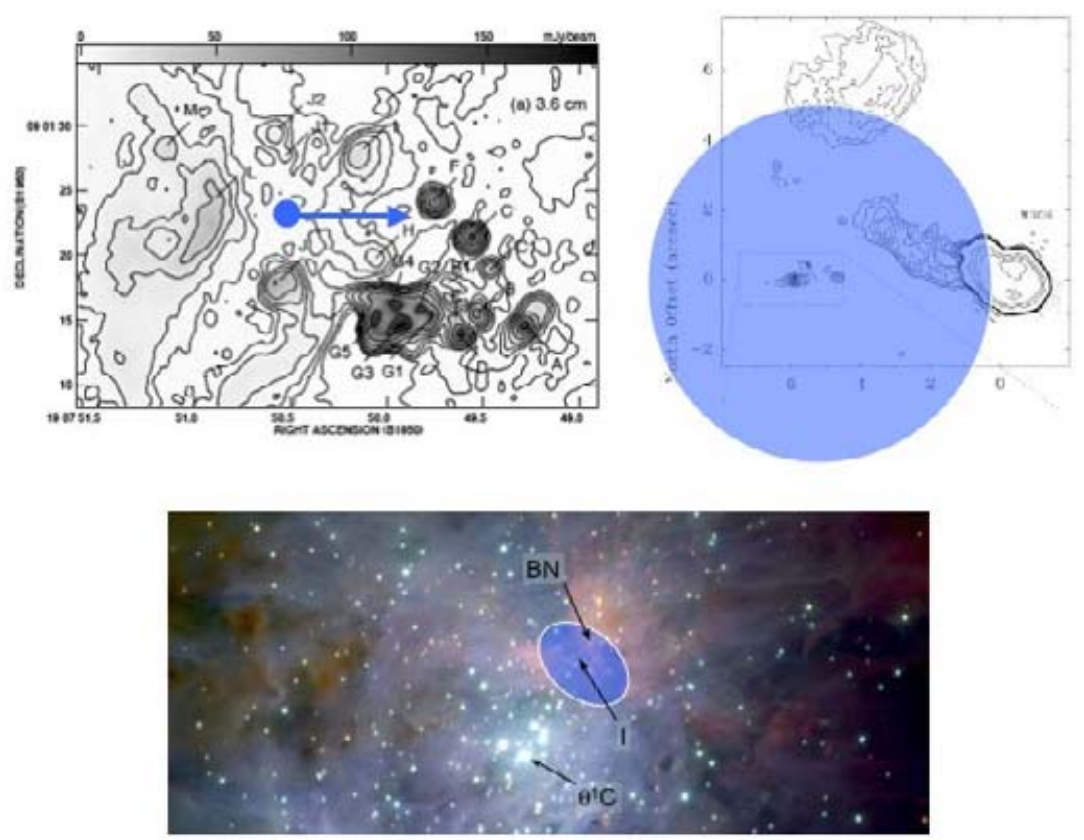

Figure 2. Comparison of the Turbulent Core Model (MT03) for the initial conditions of a $30 M_{\odot}$ star, which forms from a $60 M_{\odot}$ core, with three well-studied regions of massive star formation. The mean core radius is $R_{\text {core }} \simeq 0.06\left(M_{\text {core }} / 60 M_{\odot}\right)^{1 / 2} \Sigma^{-1 / 2}$ pc. In all cases we set $\Sigma=1 \mathrm{~g} \mathrm{~cm}^{-2}$ (i.e. the core is in pressure equilibrium with the central regions of a clump with this mean surface density). The different regions are: (a) top left the Welch ring of ultracompact HII regions in W49A shown in $3.6 \mathrm{~cm}$ emission (De Pree et al. 1997) - core location here is arbitrary, while the adjacent arrow shows the distance the core moves traveling at $\sim 5 \mathrm{~km} \mathrm{~s}^{-1}$ during its collapse time of $\sim 10^{5} \mathrm{yr}$; (b) top right $\mathrm{W} 3\left(\mathrm{H}_{2} \mathrm{O}\right)$ and $\mathrm{W} 3(\mathrm{OH})$ region viewed at $3.6 \mathrm{~cm}$ (Wilner et al. 1999) - the core is centered around $\mathrm{W} 3\left(\mathrm{H}_{2} \mathrm{O}\right)$, as suggested by the observations of Wyrowski et al. (1999); (c) bottom Orion Trapezium and BN-KL shown in the near IR (VLT-ESO \& M. McCaughrean) - the core is centered on source $I$ (Menten \& Reid 1995) and elongated slightly to match the orientation of the observed sub-mm core (Wright et al. 1992).

(1999). We conclude that the turbulent core model works reasonably well in $\mathrm{W} 3\left(\mathrm{H}_{2} \mathrm{O}\right)$. There are two radio continuum sources in the core separated by just over $0.01 \mathrm{pc}$. If these are powered by different stars and neglecting the possibility of projection effects, then two stars are forming from the same core.

\subsection{Orion $K L$}

The closest massive protostar is thought to be radio source $I$ (Menten \& Reid 1995) in the Orion Kleinmann-Low (KL) region, $\sim 450$ pc away. This region is near the center of the Orion Nebula Cluster (ONC), marked by the Trapezium OB stars (Fig. 2). Also nearby is the Becklin-Neugebauer $(\mathrm{BN})$ object, known to have a high proper motion, equivalent to about $40 \mathrm{~km} \mathrm{~s}^{-1}$ in the plane of the sky (Plambeck et al. 1995).

\subsubsection{The Ejection of $B N$}

BN's luminosity is $\sim 2500-10^{4} L_{\odot}$ (Gezari, Backman, \& Werner 1998), corresponding to a zero age main sequence B3-B4 (8-12 $\left.M_{\odot}\right)$ star. It is highly likely that BN originated in the ONC. Since the cluster is too young for binary supernova ejections, the most 
plausible model for BN's motion is dynamical ejection from an unstable triple or higher order system. This can often occur when a hard binary interacts with another star (Hut \& Bahcall 1983). Typically the least massive star is ejected at about the escape speed from the remaining binary at the orbit of the secondary, which is often left eccentric.

We have proposed BN was ejected from an interaction with the $\theta^{1} C$ system (Tan 2004c) because: (1) $\theta^{1} C$ lies along BN's past trajectory; (2) $\theta^{1} C$ has a proper motion direction opposite to BN's (van Altena et al. 1988); (3) $\theta^{1} C$ has a proper motion amplitude that would predict BN's mass is $6.4 \pm 3 M_{\odot}$, in agreement with the estimate from its luminosity; (4) $\theta^{1} C$ has a relatively massive $\left(\gtrsim 6 M_{\odot}\right)$ secondary companion (Schertl et al. 2003); (5) the projected separation of the $\theta^{1} C$ binary (total mass $\simeq 50 M_{\odot}$ ) is $17 \mathrm{AU}$ and the escape speed from this distance is $70 \mathrm{~km} \mathrm{~s}^{-1}$, high enough to explain BN's speed. To have all of the above occur by chance is highly improbable. Furthermore, no other revealed, massive ONC stars have any of the correct proper motion or binary properties.

Rodriguez et al. (2005, hereafter R05) measured proper motions of BN and source I relative to a quasar $1.6^{\circ}$ away. Previous measurements were of $\mathrm{BN}$ relative to source $I$, with the latter assumed to be a massive protostar with a motion typical of the ONC. R05 confirm the relative motion of $\mathrm{BN}$ and source $I$, but find that source $I$ is moving at $5.6 \pm 0.7 \mathrm{mas} / \mathrm{yr}\left(12 \pm 2 \mathrm{~km} \mathrm{~s}^{-1}\right)$ towards P.A. $+141^{\circ} \pm 7^{\circ}$, i.e. away from BN. R05 argue that source $I$ ejected $\mathrm{BN}$, which creates problems for the interpretation that source $I$ is a massive protostar forming from an accretion disk (see below). However, source $I$ s motion is roughly parallel to the Galactic plane and such motions would not be unreasonable for the entire ONC due to Galactic differential rotation. R05 also measure motions of 4 other nearby radio sources thought to be ONC members, reporting motions $\lesssim 3$ mas/yr relative to the quasar. However, these sources are weak and undetected at some epochs. From deviations from straight line motion we estimate positional uncertainties of $\gtrsim 50$ mas in some sources, leading to motion errors of $\gtrsim 4$ mas/yr. We conclude the data are not yet good enough to tell if source $I$ has a motion atypical of the ONC.

\subsubsection{Source I: Core, Disk, Protostar and Outflow}

Wright et al. (1992) mapped a core of dense gas in the KL region in emission at $450 \mu \mathrm{m}$. This core is about a factor of two smaller than that shown in Fig. 2, as might result from the contraction that occurs after the onset of collapse. The polarization vectors of near IR emission suggest that a single source is responsible for much of the luminosity from the core (Werner, Capps, \& Dinerstein 1983), and this source is likely to be the thermal radio source $I$ (Menten \& Reid 1995), which lies near the center of the core.

Wright et al. (1995), Greenhill et al. (1998) and Tan (2004c) have interpreted the system as containing a $r \sim 1000 \mathrm{AU}$ accretion disk, as traced by $\mathrm{SiO}(\mathrm{v}=0 ; \mathrm{J}=2-1)$ maser emission, centered about source $I$. This scale is similar to that estimated from collapse of a core with $\beta=0.02$ (§3.3). The velocity of maser spots from different sides of the disk suggest a central mass of about $20 M_{\odot}$. The disk alignment is perpendicular to the large scale molecular outflow to the NW and SE (Chernin \& Wright 1996).

The luminosity of source $I$ (we equate this to the region often referred to as the "Orion hot core") is $1.3-5 \times 10^{4} L_{\odot}$ (Kaufman et al. 1998). MT03 used this to estimate a current protostellar mass of $11-18 M_{\odot}$ and accretion rate $2.2-5.0 \times 10^{-4} M_{\odot} \mathrm{yr}^{-1}$. The corresponding H-ionizing photon luminosities are $10^{45}-10^{48} \mathrm{~s}^{-1}$ (Tan \& McKee 2002).

Tan \& McKee (2003) modeled the HII region produced when an ionizing source, i.e. a massive protostar, is embedded at the base of a neutral disk wind or $\mathrm{X}$-wind. This "outflow-confined", hyper-compact HII region model appears to work well for source I: one model that can explain the radio spectrum has an ionizing photon luminosity of $2 \times 10^{47} \mathrm{~s}^{-1}$ and a mass outflow rate of $3 \times 10^{-5} M_{\odot} \mathrm{yr}^{-1}$, about $10 \%$ of the estimated 
accretion rate. Source $I$ is elongated (Menten \& Reid, in prep.), and the position angle of elongation aligns well with a Herbig-Haro object to the NW (Taylor et al. 1986) requiring flow velocities $\sim 1000 \mathrm{~km} \mathrm{~s}^{-1}$, which is about the escape speed from a $20 M_{\odot}$ protostar. $\mathrm{SiO}(\mathrm{v}=1 \& 2)$ masers have been observed immediately surrounding the radio source on scales of several tens of AU (Greenhill et al. 2003). The densities and temperatures of the gas in the outflow-confined HII region model are appropriate for the excitation of these masers. The velocities of these masers are rather low $\left(\sim 10-20 \mathrm{~km} \mathrm{~s}^{-1}\right)$ and exhibit a gradient along the elogated direction of source I. Greenhill et al. (2003) used this to argue that the disk is in fact orientated along this axis, perpendicular to previous disk models. However, there is little evidence for a large outflow in the direction expected for this orientation and a new source would be needed for the powerful NW-SE outflow.

In conclusion, many aspects of the Orion KL region and source $I$ can be explained by the Turbulent Core Model for massive star formation. The scale of the core is relatively large, meaning that, in the context of this model, it is not possible to form numerous massive stars in this region. The assumption that the massive star forms in isolation is obviously an approximation: the accretion flow can suffer perturbations from the close passage of other stars, but only relatively massive stars, such as BN, will have a potentially significant effect. Other stars are likely to be forming near the collapsing massive core, but the opportunity for fragmentation from the core itself is limited because it is globally collapsing on a relatively short timescale, i.e. approximately its free-fall timescale. The difference between this case and star clusters, which do fragment into mostly low-mass stars, is probably the collapse time: rich star clusters are likely to take many free-fall timescales to form (Tan 2004a).

\section{Acknowledgements}

I thank many colleagues for discussions, particularly Chris McKee, Mark Krumholz, and Luis Rodriguez. I am supported by a Zwicky fellowship from ETH.

\section{References}

Alves, J. F. Lada, C. J., \& Lada, E. A. 2001, Nature, 409, 159

Bally, J., \& Zinnecker, H. 2005, AJ, 129, 2281

Bate, M. R., Bonnell, I. A., \& Price, N. M., 1995, MNRAS, 277, 362

Beuther, H., \& Schilke, P. 2004, Science, 303, 1167

Bonnell, I. A., Bate, M. R., \& Zinnecker, H. 2004, MNRAS, 298, 93

Bonnell, I. A., Bate, M. R., Clarke, C. J., \& Pringle, J. E. 2001, MNRAS, 323, 785

Bonnell, I. A., Vine, S. G., \& Bate, M. R. 2004, MNRAS, 349, 735

Boulares, A., \& Cox, D. P. 1990, ApJ, 365, 544

Carey, S. J., Clark, F., Egan, M., Price, S., Shipman, R., \& Kuchar, T. 1998, ApJ, 508, 721

Chernin, L. M., \& Wright, M. C. H. 1996, ApJ, 467, 676

Crutcher, R. M. 1999, ApJ, 520, 706

De Pree, C. G., Mehringer, D. M., \& Goss, W. M. 1997, ApJ, 482, 307

de Wit, W. J., Testi, L., Palla, F., \& Zinnecker, H. 2005, A\&A, 437, 247

Deharveng, L., Zavagno, A., \& Caplan, J. 2005, A\&A, 433, 565

Dobbs, C. L., Bonnell, I. A., \& Clark, P. C. 2005, MNRAS, 360, 2

Edgar, R., \& Clarke, C. 2004, MNRAS, 349, 678

Elmegreen, B. G. 2000, ApJ, 530, 277

Elmegreen, B. G. 2004, in The Formation and Evolution of Massive Young Star Clusters, ed. H. Lamers et al., ASP 322, 277

Elmegreen, B. G., \& Lada, C. J. 1977, ApJ, 214, 725

Gezari, D. Y., Backman, D. E., \& Werner, M. W. 1998, ApJ, 509, 283

Gilbert, A. M., \& Graham, J. R. 2003, in Extragalactic Globular Clusters, 25th IAU, JD 6 
Goodman, A. A., Benson, P. J., Fuller, G. A., \& Myers, P. C. 1993, ApJ, 406, 528

Goodman, J., \& Tan, J. C. 2004, ApJ, 608, 108

Greenhill, L. J., Gwinn, C. R., Schwartz, C., Moran, J., \& Diamond, P. 1998, Nature, 396, 650

Greenhill, L. J., Reid, M. J., Chandler, C. J., Diamond, P., \& Elitzur, M. 2003, IAU Symp. 221, Star Formation at High Angular Resolution, eds. Burton et al., ASP, (astro-ph/0309334)

Hachisuka, K., Brunthaler, A., Hagiwara, Y., Menten, K. M., Imai, H., Miyoshi, M., \& Sasao, T. 2004, 7th European VLBI Network Symp., eds. Bachiller et al., (astro-ph/0409343)

Hut, P., \& Bahcall, J. N. 1983, ApJ, 268, 319

Kaufman, M. J., Hollenbach, D. J., \& Tielens, A. G. G. M. 1998, ApJ, 497, 276

Kim, S. S., Figer, D. F., Lee, H. M., \& Morris, M. 2000, ApJ, 545, 301

Klessen, R., Ballesteros-Paredes, J, Vázquez-Semadeni, E, \& Durán-Rojas, C. 2005, ApJ,620,786

Krumholz, M. R., McKee, C. F., \& Klein, R. I. 2005, ApJ, 618, 757

Lada, C. J., \& Lada, E. A. 2003, ARA\&A, 41, 57

Larson, R. B. 2005, MNRAS, 359, 211

Mac Low, M., \& Klessen, R, S. 2004, Reviews of Modern Physics, 76, 125

McLaughlin, D. E., \& Pudritz, R. E. 1997, ApJ, 476, 750

McKee, C. F. 1989, ApJ, 345, 782

McKee, C. F. 1999, in NATO ASIC Proc. 540: The Origin of Stars and Planetary Systems, p29.

McKee, C. F., \& Tan, J. C. 2002, Nature, 416, 59

McKee, C. F., \& Tan, J. C. 2003, ApJ, 585, 850 (MT03)

Mengel, S., Lehnert, M. D., Thatte, N., \& Genzel, R. 2002, A\&A, 383, 137

Menten, K. M., \& Reid, M. J. 1995, ApJ, 445, L157

Mouschovias, T. Ch. 1996, Solar and Astrophysical MHD Flows, ed. Tsiganos, (Kluwer), 505

Mueller, K. E., Shirley, Y. L., Evans, N. J., \& Jacobson, H. R. 2002, ApJS, 143, 469

Oey, M. S., Watson, A. M., Kern, K., \& Walth, G. L. 2005, AJ, 129, 393

Osorio, M., Lizano, S., \& D'Alessio, P. 1999, ApJ, 525, 808

Palous, J., Tenorio-Tagle, G., \& Franco, J. 1994, MNRAS, 270, 75

Plambeck, R. L., Wright, M. C. H., Mundy, L. G., \& Looney, L. W. 1995, ApJ, 455, L189

Rodriguez, L. F., Poveda, A., Lizano, S., \& Allen, C. 2005, ApJ, 627, L65 (R05)

Schertl, D., Balega, Y. Y., Preibisch, Th., \& Weigelt, G. 2003, A\&A, 402, 267

Schmeja, S., \& Klessen, R. S. 2004, A\&A, 419, 405

Scoville, N. Z., Sanders, D. B., \& Clemens, D. P. 1986, ApJ, 310, L77

Solomon, P. M., Rivolo, A. R., Barrett, J., \& Yahil, A. 1987, ApJ, 319, 730

Shu, F. H., Adams, F. C., \& Lizano, S. 1987, ARA\&A, 25, 23

Tan, J. C. 2000, ApJ, 536, 173

Tan, J. C. 2004a, in The Young Local Universe, (astro-ph/0407093)

Tan, J. C. 2004b, in Star Formation in the Interstellar Medium, ASP, 323, 249

Tan, J. C. 2004c, ApJ, 607, L47

Tan, J. C., \& McKee, C. F. 2002, in Hot Star Workshop III: Massive Star Birth, ASP 267, 267

Tan, J. C., \& McKee, C. F. 2003, IAU Symp. 221, Star Formation at High Angular Resolution, eds. Burton et al., ASP, (astro-ph/0309139).

Taylor, K., Dyson, J. E., Axon, D. J., \& Hughes, S. 1986, MNRAS, 221, 155

Thompson, T. A., Quataert, E., \& Murray, N. 2005, ApJ, in press, (astro-ph/0503027)

Turner, J. L., Beck, S. C., \& Ho, P. T. P. 2000, ApJ, 532, L109

van Altena, W. F., Lee, J. T., Lee, J.-F., Lu, P. K., \& Upgren, A. R. 1988, AJ, 95, 1744

Vázquez-Semadeni, E., Kim, J., Shadmehri, M., \& Ballesteros-Paredes, J. 2005, ApJ, 618, 344

Walsh, A. J., Myers, P. C., \& Burton, M. G. 2004, ApJ, 614, 194

Werner, M. W., Capps, R. W., \& Dinerstein, H. L. 1983, ApJ, 265, L13

Whitworth, A. P., \& Francis, N. 2002, MNRAS, 329, 641

Wilner, D. J., Reid, M. J., \& Menten, K. M. 1999, ApJ, 513, 775

Wright, M. C. H., Plambeck, R. L., Mundy, L. G., \& Looney, L. W. 1995, ApJ, 455, L185

Wright, M. C. H., Sandell, G., Wilner, D. J., \& Plambeck, R. L. 1992, ApJ, 393,225

Wyrowski, F., Schilke, P., Walmsley, C. M., \& Menten, K. M. 1999, ApJ, 514, L43

Yorke, H. W., \& Sonnhalter, C. 2002, ApJ, 569, 846 